\title{
HACIA UNA CONSTRUCCIÓN DE CIUDADANÍA AMBIENTAL EN LA ESCUELA
}

\author{
MARTHA CECILIA RODRÍGUEZ PINZÓN \\ Licenciada en Biología, Especialista en Auditoría y Estudios Ambientales. \\ Estudiante de Maestría en Educación, Universidad del Tolima. \\ maceropi1@hotmail.com
}

\begin{abstract}
Cómo citar este artículo:
Rodríguez, M. (2011) La ética: Punto de encuentro de la educación ambiental y el ser profesional. Espiral, Revista de Docencia e Investigación. 1, (1), 13 - 20.
\end{abstract}

\begin{abstract}
Resumen
A partir de una refexión sobre la creciente degradación ambiental como producto de las relaciones sociales irresponsables e irrespetuosas con el medio ambiente, se concibe la salida de esta crisis a través de procesos de Educación ambiental en la formación para la ciudadanía. Se incluyen, en este artículo, antecedentes sobre Educación ambiental ciudadana en los últimos años, los cuales conllevan a plantear que la construcción de unas relaciones humanas sensibles, respetuosas, solidarias y responsables con el medio ambiente, se pueden propiciar en todos los contextos y situaciones de la vida escolar, constituyéndose en un reto: ¿Cómo articular y promover las competencias de la Educación Ambiental con las de formación ciudadana conducentes al fortalecimiento de un pensamiento ambiental en la escuela, hacia la construcción de una ciudadanía ambiental?
\end{abstract}

Palabras clave: Medio ambiente, ciudadanía, ética, estética, competencia, pensamiento ambiental.

\begin{abstract}
From a refection on the increasing environmental degradation as a result of irresponsible and disrespectful social relations with the environment is conceived as a solution to this crisis, environmental education in the process of training for citizenship. In this article are included background on environmental education in citizenship training in recent years, which lead to pose that the construction of humans relation, sensitive, respectful, caring and responsible with the environment, can be facilitated in all contexts and situations of school life, becoming a challenge ¿How to articulate and promote environ-
\end{abstract}

mental education competencies with competencies of citizenship training, to strengthen environmental thinking in the school towards the construction of an environmental citizenship?

Keywords: environment, citizenship, ethics, aesthetics, competition, environmental thinking.

\section{Introducción}

La problemática ambiental es, en gran parte, una consecuencia de carácter eminentemente humana generada por procesos culturales y sociales insustentables, no amigables con el medio ambiente. Por lo mismo, los cambios y soluciones deben darse en la cultura social que ha causado el deterioro de la base natural y es la educación ambiental la llamada a generar estas transformaciones desde la escuela.

Frente a esta posición, Telias (2010), establece que la crisis ambiental exige una educación ambiental comprometida con el ejercicio de una ciudadanía que tiene una responsabilidad impostergable frente a la defensa del derecho a un ambiente sano y, por ello, es prioritaria una participación ciudadana activa; en este enfoque, la educación tiene un rol primordial, pues desafortunadamente el sistema educativo, obediente a las 
políticas de los Estados y a la economía capitalista reinante ha contribuido a cimentar esta problemática ambiental.

Es a partir de los procesos de socialización en la escuela, desde los niños y jóvenes en formación, donde puede iniciarse la apropiación de unas relaciones sensibles, armoniosas y responsables entre sujeto-medio ambiente-sociedad, a través de un nuevo pensamiento ambiental, hacia la
La problemática ambiental es, en gran parte, una consecuencia de carácter eminentemente humana generada por procesos culturales y sociales insustentables, no amigables con el medio ambiente construcción de una ciudadanía que conduzcan a una verdadera sustentabilidad y a la conservación del entorno natural y por ende, a un ambiente sano y de vida para el ser humano.

Frente a lo anterior, la Educación Ambiental es la convocada a generar estos cambios y la transformación de concepciones, saberes y quehaceres a través de su aparición en la historia. Sin embargo, ha sido una tarea desarticulada con las otras ciencias, inclusive de las Ciencias Naturales y de las Ciencias Sociales tan fundamentales en estos procesos. Las Sociales que no conectan las actuaciones de la sociedad con sus repercusiones en la naturaleza; las Naturales que no han llevado a refexionar sobre las relaciones sistémicas complejas de la naturaleza y los impactos de las relaciones sociales, la cultura, la ciencia y la tecnología sobre la trama de la vida en los ecosistemas. Por consiguiente, los esfuerzos y las propuestas mundiales, nacionales y regionales dadas, alejadas de una visión ecosistémica de la naturaleza, unidas a la ausencia de una ética ambiental, no han tocado frmemente el pensamiento, la conciencia y la responsabilidad humana. Así, el deterioro y el uso irracional de los recursos naturales continúan.

De igual manera, las instituciones educativas no escapan a esta situación. El diagnóstico que presentan los Ministerios del Medio Ambiente y de Educación (2002) en la Propuesta de la Política Nacional de Educación Ambiental frente a la problemática ambiental en el sector educativo formal colombiano de básica y media, cuyos resultados persisten en la actualidad, pone en evidencia la descontextualización, descoordinación y desarticulación en las acciones que lleva a cabo la escuela desde sus mismos proyectos pedagógicos, frente a las competencias y responsabilidades tanto en la formación de ciudadanía como en los procesos de educación ambiental, lo que ha traído como consecuencia la improvisación, el activismo, la inmediatez y la escasez de cambios positivos responsables. Se suma la carencia de una verdadera formación ciudadana en mecanismos de participación relacionados con la problemática y las diversas dinámicas ambientales, lo que difculta la apropiación social del entorno ambiental.

La problemática ambiental no es algo puntual que se soluciona con recetas mecanizadas e instrumentales en un sitio y tiempo limitados. "Existe un activismo inmediatista que conlleva a realizar medidas que aparentemente solucionan problemas concretos, de manera inmediata. Son actividades ciegas que evaden un análisis sistémico, complejo o preguntas de fondo" (Eschenhagen 2007,16) y no originan soluciones de fondo.

Frente a esta situación crítica ambiental en la escuela, en el contexto local, nacional y planetario, reconociéndola como producto de las relaciones culturales insustentables con el entorno natural, es pertinente y necesario, dar una mirada a algunas propuestas y procesos que están avantes y en perspectiva, frente a los procesos de educación ambien- 
tal en la formación para la ciudadanía, que ofrezcan luces a seguir en el contexto escolar particular, para avanzar en una propuesta pedagógica que dé salida, freno o reducción al deterioro ambiental.

Al respecto, en España, Novo y Murga (2010) en "Educación Ambiental y ciudadanía planetaria" parten de la concepción de ciudadanía como el resultado de la relación entre los individuos con su comunidad, ubicándose en ella y sintiéndose parte activa de la misma, con derechos y deberes construcción de una ciudadanía planetaria sostenible, basada en la capacidad de asombro ante la vida
El reto de la y con un sentido de pertenencia. Proponen unas pautas para que la Educación Ambiental frente a la actual sociedad compleja y globalizada, tenga el reto de la construcción de una ciudadanía planetaria sostenible, basada en la capacidad de asombro ante la vida, la moderación y precaución en el uso de los recursos, el desarrollo de valores en lo asociativo, la solidaridad, el respeto al otro, la pluralidad, la diversidad ecológica y cultural, la comprensión recíproca y la tolerancia, conscientes de la ecodependencia, comprometiéndose con el cuidado y la preservación de la vida en todas sus expresiones.

El anterior enfoque muestra a través de una refexión histórica de la Educación Ambiental, cómo el cambio se puede dar a través del fortalecimiento integral de las relaciones humanas con su entorno natural, desde lo cognoscitivo, afectivo, axiológico y estético. Frente a esta exigencia, la escuela se torna en escenario ideal para favorecer el desarrollo de estos procesos, lo que le exige un replanteamiento creativo de una nueva pedagogía que busque interiorizar el respeto, el uso responsable de los recursos naturales, el cuidado y la conservación de la vida humana y la biodiversidad.
Continuando con esta mirada hacia Norteamérica, Houser, N. (2009) en su artículo investigativo "Ecological democracy: An environmental approach to citizenship education" publicada en Theory and research in social education aporta el concepto de "democracia ecológica" como la participación activa-positiva de los ciudadanos con la naturaleza, poseedores de una sensibilidad ecológica que los conduce a prácticas basadas en la responsabilidad cívica, en un frme y permanente compromiso frente al mantenimiento de la paz, la democracia y un sistema ecológicamente viable para la vida. Proyecta su enseñanza en la escuela a través del plan de estudios con la participación comprometida de los docentes en todas las áreas, donde su aprendizaje, sea un objetivo fundamental soportado por principios: conocimiento, el respeto mutuo, pluralidad, empatía, diversidad social, cultural y ambiental. La búsqueda de alternativas de solución como un proyecto creativo, de fuerte apropiación, colaboración y participación activa de docentes y estudiantes.

Sin embargo, la Democracia Ecológica, como la enfoca Houser, no es conveniente matricularla como una temática o asignatura más, pues la experiencia de los resultados del enfoque por asignaturas, pone en evidencia el riesgo de sesgarla, desarticularla y de perder su importancia e interés. Construirla entonces, como un proceso transversalizado, vivencial, refexivo, participativo, dentro del marco de experiencias signifcativas que abarquen la vida cotidiana escolar.

En este contexto, desde Argentina, Alvino, Canciano, Sesano, Telias y Sessano (2007), en su artículo científco "Construcción ciudadana y educación ambiental: derechos, ambiente y ciudadanía", enmarcado en su trabajo de investigación educativa "Emergencia del campo de la educación ambiental en Argentina: historia de sus ideas 
fundantes e inscripción de las experiencias en las alternativas pedagógicas de América Latina" de la Universidad de Argentina, plantean que la concepción de ambiente frente a la construcción de ciudadanía, implicaría verlo como un derecho inherente a la democracia, una complejidad y un compromiso humano frente a la crisis ambiental.

Desde este enfoque, se establece que la vida, la naturaleza y el ser humano poseen el derecho a un ambiente sano y diverso que necesariamente conlleva a un tipo de relaciones, de prácticas culturales para la preservación de la vida natural en todas sus manifestaciones, y a la apropiación de condiciones, estrategias $\mathrm{y}$ acciones sustentables que lo garanticen en el tiempo y en el espacio. Esta posición exige que dichas relaciones y prácticas culturales, transversalicen los diferentes proyectos y actividades en los distintos contextos sociales cotidianos de la escuela llevando a fortalecer la educación ambiental en la construcción de una ciudadanía "como un proceso colectivo... donde la participación activa y comprometida con la trama socioambiental y política de la cultura quede enmarcada en una concepción compleja de ambiente..." $(2007,153)$

Esta concepción del ambiente se distancia de antropocentrismo imperante que en aras de un desarrollo económico y una calidad de vida falaz, ha tomado dominio y explotación de los recursos naturales, al romper el equilibrio de los ecosistemas, llevándolos a límites peligrosos de afectación. Visión que ha ejercido infuencia en el énfasis educativo ambiental que aún se mantiene en las escuelas: el de la concepción del ambiente como fuente inagotable de recursos naturales.

\section{Pensamiento refexivo}

Paralelamente a la exigencia de estos cambios, surge imprescindible para el desarrollo de estos procesos, la construcción de un pen- samiento refexivo sobre el ambiente, desde una "ética-estética ambiental" como la plantea Noguera (2004) en su libro "El reencantamiento del mundo: ideas flosófcas para la construcción de un pensamiento ambiental contemporáneo". Una ética sistémica, donde la compleja trama de la vida de la cual hace parte el ser humano, pasa de ser objeto de dominio, de uso irracional, a ser un valor moral en el cual prevalecen la solidaridad, la cooperación, el respeto y la responsabilidad. Una estética que retorne al "reencantamiento del mundo", a la contemplación, la sensibilidad, la creatividad y las artes.

De acuerdo con Noguera (2007) en su artículo "Complejidad ambiental: propuestas éticas emergentes del pensamiento ambiental latinoamericano", pensadores latinoamericanos ambientales como: Augusto Ángel Maya, Guillermo Hoyos, Julio Carrizosa, Daniel Vidart, Arturo Escobar, Rubiel Ramírez, Enrique Leff y Leonardo Boff, a través de su pensamiento ético-ambiental coinciden en que los valores de la responsabilidad, el respeto, la solidaridad, la cooperación, la democracia y diversidad cultural, se constituyen en principios y bases de una ética ambiental. Idea en la que convergen la gran mayoría de las propuestas en el campo de la gestión y de la educación ambiental.

No obstante, la mirada de un gran número de autores y proyectos en esta área, conducen hacia el desarrollo sostenible. Posición controvertida en la actualidad, fuertemente criticada por estos pensadores latinoamericanos que lo tildan de "capitalismo pintado de verde", pues lo consideran una herramienta de la economía globalizada abrasiva para saquear y explotar el entorno natural, mantener su poder y benefcio, con muy pocos avances en el freno de la degradación y calentamiento global del planeta.

Desde esta perspectiva, la educación ambiental hacia el desarrollo sostenible su- 
pone una postura individualista, de control económico, que no puede alejarse de su fundamento consumista y no evidencia logros signifcativos, ni logrará frenar o reducir los males del medio ambientesociedad, pues se sigue interviniendo sin considerar la complejidad en la que se sustentan los ecosistemas. Por lo mismo, es imprescindible

“cambiar las relaciones entre las culturas y los ecosistemas. Mientras la mirada a esas relaciones entre los ecosistemas y la cultura sea una mirada de dominio y los discursos del desarrollo, aún del desarrollo sostenible, sigan imperando, seguiremos siendo una especie ingrata, y morando la tierra - ese mundo de la vida simbólico-biótico del cual hacemos parte - como si fuera una bodega llena de recursos disponibles y para siempre". (Noguera, 2004, 47)

El llamado, entonces, es que las propuestas ambientales $y$, en especial, las de educación ambiental deben llevar al desarrollo de un "pensamiento ambiental", fundamentado en una concepción, no de explotación industrial irracional, ni de poder económico del ambiente disfrazado de ecologismo y sustentabilidad, cuyos resultados son la contaminación, el agotamiento de los recursos naturales y el desmejoramiento de la calidad de vida de muchos, sino en un aprovechamiento y manejo respetuoso y responsable del equilibrio complejo del mundo natural.

Esta mirada, le ofrece otra perspectiva a la educación ambiental en la cultura escolar, pues orienta a plantear, más que prácticas ambientales que sólo conduzcan a la puntualidad e inmediatez de soluciones temporales, una articulación de saberes y acciones desde la dimensión ambiental que transversalicen el mundo de la escuela; la conexión, comprensión y refexión de las relaciones ecosistémicas, transformación de actitudes, fortalecimiento de valores y la apropiación de habilidades que fortalezca en los estudiantes, un "pensamiento ambiental" fundamentado en una concepción no material del ambiente, por fuera del consumismo y el dominio, que lleve a relacionar de manera armoniosa y sensible los ecosistemas y las culturas. A investigar, buscar otras maneras de descubrir, conocer, pensar, obrar, aprovechar y de habitar el planeta tierra. Esto exige un replanteamiento de las relaciones sociales en la escuela frente a su entorno natural, no por imposición sino por goce y convicción; retornar a la estética, despertar las fbras sensibles y humanas de cada estudiante; volverlo al disfrute del contacto armonioso y respetuoso de la naturaleza y la vida misma.

En esta corriente del pensamiento ambiental, se traen los aportes de la tesis de grado de Maestría en

Medio Ambiente y desarrollo de la Universidad Nacional

$$
\begin{aligned}
& \text { "La Escuela } \\
& \text { como escenario } \\
& \text { de compleji- } \\
& \text { dad (La Educa- } \\
& \text { ción Ambien- } \\
& \text { tal desde la } \\
& \text { complejidad)" }
\end{aligned}
$$
de Colombia: "La Escuela como escenario de complejidad (La Educación Ambiental desde la complejidad)" de Londoño (2008) quien concibe que la educación ambiental, debe dejar el reduccionismo y la fragmentación y tener en cuenta el conjunto de relaciones e interdependencias entre las condiciones naturales y las culturales. Formula una propuesta de educación ambiental como un proceso incluyente, un proceso cognitivo, un proceso de conocer los lenguajes de la naturaleza y pensar evolutivamente. Manifesta la urgencia de prácticas creativas y refexivas que aporten a la pedagogía, la compasión, la solidaridad, que permita y promueva espacios para el diseño de una nueva sociedad a través de los proyectos pedagógicos transversales que promuevan 
espacios democráticos, bioéticos, estéticos desde lo ambiental, en una labor institucional conjunta y comprometida.

Conjuntamente, es oportuno citar las propuestas ministeriales de la Educación Ambiental y formación para la ciudadanía por competencias en Colombia. Desde esta mirada, el Ministerio de Educación Nacional (2006) formuló una propuesta: "Estándares básicos de competencias ciudadanas. Formación para la ciudadanía", en busca del desarrollo de "Estándares seres humanos compebásicos de tentes para posibilitar competencias ciudadanas.

Formación para la ciudadanía" las relaciones humanas y la acción constructiva del estudiante en la sociedad, mediante la formación ciudadana en la educación básica y media, a través de competencias ciudadanas integradoras: comunicativas, cognitivas y emocionales, que fomenten el desarrollo moral, aporten a la construcción de la convivencia, promuevan la democracia, la pluralidad y el respeto por diversidad, la identidad y valoración de las diferencias humanas, la tolerancia y la solidaridad.

Como se percibe, esta propuesta de formación ciudadana proyecta abarcar todos los espacios de la escuela donde se manifesten relaciones humanas y favorece escenarios de participación y concertación educativa en aras del fomento de la democracia escolar que estaba y continúa en la actualidad, centrada en el desarrollo de actividades en torno al gobierno escolar. Sin embargo, las competencias están centradas en el ser humano como el todo y no como parte del entramado complejo de su entorno natural, desarticulando las relaciones sociales de las relaciones ecosistémicas, como si la naturaleza fuera algo por fuera del hombre.
En el panorama ambiental el Ministerio de Educación Nacional y Ministerio del Medio Ambiente (2002) desde la propuesta de la Política Nacional de Educación Ambiental en Colombia propone una visión dirigida hacia la construcción de una cultura ambiental ética, responsable frente al manejo de la vida en todas sus formas y en general frente al manejo del ambiente; respetuosa de la diversidad ecológica y cultural, para lo cual, la sostenibilidad de los contextos naturales y sociales son un reto. Para ello, institucionaliza la educación ambiental en la educación básica y media y establece los proyectos educativos ambientales escolares (Praes).

A pesar de ser una propuesta que ha sido reconocida mundialmente por autoridades y expertos del mundo por su fundamentación investigativa y avances en materia de institucionalización en los sectores educativo y ambiental del país, si bien, se han dado esfuerzos y buenas intenciones, la realidad actual en la escuela y en la comunidad no evidencia alcances realmente signifcativos en materia de Educación ambiental.

Frente a esta situación, Torres (2008) establece una refexión sobre las difcultades que presentan los Proyectos ambientales escolares en Colombia, frente a su incidencia en el quehacer de las comunidades y menciona que se debe en gran parte a su desarticulación integral con los Proyectos Educativos Institucionales (PEI), el desconocimiento de las relaciones sociales y ecosistémicas y la falta de estrategias pedagógicas signifcativas que busquen la articulación transversal e interdisciplinaria de los Proyectos ambientales escolares (Praes) con la vida cotidiana de la institución educativa y la comunidad circundante.

Para buscar un común denominador entre las dos propuestas ministeriales, se impone entonces, la formación de nuevos ciudadanos con capacidad refexiva para reconocerse, no 
como el centro sino como parte integral del ambiente, responsable de sus problemáticas y de sus posibles soluciones; hábiles en la búsqueda de consensos para la resolución de confictos y con un alto sentido de pertenencia con su medio natural y social.

De igual forma, la propuesta de Houser (2009) encuentra elementos comunes con los lineamientos de la Propuesta Nacional de Educación ambiental (MEN, 2002) y plantea que una forma de llegar a establecer relaciones armoniosas sostenibles entre sujeto social-naturaleza y frenar la degradación ambiental es a través de la construcción de un proyecto de ciudadanía con miras hacia lo ambiental, donde converjan las competencias y criterios de la formación cívica ciudadana con los principios y criterios de la Educación ambiental.

\section{A manera de conclusión}

Es por ello, que los enfoques y las propuestas ambientales de los diferentes autores citados en esta refexión y la propuesta de los ministerios que orientan los procesos de formación ciudadana y educación ambiental en Colombia, aportan elementos valiosos y se
La educación ambiental, debe salirse del marco de un currículo lineal relacionan en forma directa con la construcción de una propuesta ambiental hacia lo ciudadano. La escuela de manera fexible, debe favorecer espacios para este trabajo. Esto implica comprender que la educación ambiental como la plantean los pensadores ambientales contemporáneos latinoamericanos y otros, junto a la Política Ambiental Colombiana, debe salirse del marco de un currículo lineal, fragmentado disciplinar y exige la interdisciplinariedad y la transversalidad que reconcilie los saberes, que retorne a la naturaleza, no para dominarla, explotarla o destruirla, sino orientada a tra- bajar el mundo complejo de la naturaleza como un todo, donde todas las disciplinas tanto las ciencias duras como las humanas dejen de convertir el mundo natural en pedazos y comiencen a retomarlo como un sistema integral.

Por tal motivo, el compromiso del fortalecimiento de la educación ambiental en la construcción de ciudadanía es una prioridad; exige una resignifcación de un tipo de prácticas ambientales ciudadanas y relaciones más humanas y comprometidas, que se conciben a favor de un ambiente sano y de vida. Es la escuela, en su cotidianidad, el medio propicio para promover estas competencias.

Por lo tanto, la construcción de unas relaciones humanas sensibles, conscientes, responsables y equilibradas con el medio ambiente, se pueden propiciar en todos los contextos y situaciones de la vida escolar, constituyéndose en una posibilidad y una exigencia, la articulación de competencias propias de la educación ambiental con las de formación ciudadana, para promover competencias ambientales- ciudadanas integradoras y formativas, conducentes al fortalecimiento de un pensamiento ambiental desde la comprensión y refexión de las relaciones ecosistémicas y su conexión con las relaciones sociales-culturales, fundamentada en una escala de actitudes y valores desde la ética-estética ambiental.

De modo tal que contribuyan a una educación integral responsable frente al manejo de la vida en todas su diversidad y en general frente al manejo del ambiente, para contribuir a la formación de ciudadanos ambientales escolares con sensibilidad y capacidad refexiva para identifcarse como parte integral del mundo natural, de su conservación, protección, uso racional, problemática y sus posibles soluciones, hacia la construcción de una ciudadanía ambien- 
tal que trabaje por un verdadero desarrollo sostenible, un mejoramiento de la calidad de vida y un ambiente armonioso y de vida.

Frente a lo expuesto, queda el reto de investigar una propuesta pedagógica creativa donde se plantee... ¿Cómo articular y promover las competencias propias de la Educación Ambiental con las de formación ciudadana, para fortalecer el pensamiento ambiental en la escuela, con miras a la construcción de una ciudadanía ambiental?

\section{Referencias bibliográfcas}

Alvino, S., Canciani, L., Sessano, P., y Telias, A. (2007). La ciudadanía y el derecho al ambiente: refexiones en torno a una articulación. Anales de la educación común: Educación y ambiente. 3(8), 152-161. Disponible en http://abc.gov.ar/lainstitucion/ revistacomponents/revista/archivos/anales / numero08/archivosparadescargar/19_ciudadania.pdf

Houser, N. (2009).Ecological Democracy: An environmental approach citizenship education. Theory and research in social education, 37(2), 192-214. Recuperado de http://dialnet.unirioja.es/servlet/listaarticulos?tipo_ b u s qu ed a = E J E M P L A R \& r e vis t a busqueda $=1427 \&$ clave_busqueda $=227964$

Eschenhagen, M. L. (2007) ¿Será necesario ambientalizar la educación ambiental? En Ana Noguera de Echeverry, Patricia (comp.), Hojas De Sol en la Victoria Regia. Emergencia de un Pensamiento Ambiental Alternativo en América Latina, (113-148).Manizales: Ed. Universidad Nacional.

Londoño, C. A. (2008). La escuela como escenario de complejidad (La Educación Ambiental desde la complejidad). (Tesis de Maestría, Universidad Nacional de Colombia). Recuperado de http:/ / www.bdigital.unal.edu.co/1130/1/claudiaandrealondonorestrepo.2008.pdf

Ministerio de Educación Nacional. (2006). Estándares básicos de competencias. Formación para la ciudadanía. Bogotá: MEN.

Ministerio de Educación Nacional y Ministerio del Medio Ambiente. (2002). Política Nacional de Educación Ambiental. Bogotá, Colombia.
Noguera, A.P. (2007). Complejidad Ambiental: propuestas éticas emergentes del pensamiento ambiental latinoamericano. Revista Gestión y Ambiente, 10 (1), 5-30. Recuperado de http:/ / www. revistas.unal.edu.co

(2004). El Reencantamiento del Mundo: Ideas Filosófcas para la Construcción de un Pensamiento Ambiental Contemporáneo, 55-67. Manizales: Editorial Pnuma Universidad Nacional.

Novo, M. y Murga, M. (2010). Educación Ambiental y Ciudadanía planetaria. Revista Eureka sobre Enseñanza y Divulgación de las ciencias, 7(Extraordinario), 179-186. Recuperado http://www. apac-reka.org/revista/Volumen7/Numero_7_ extra/1_Novo_Murga_2010.pdf

Ruiz-Silva, A. y Chaux, E. (2005). Formación ciudadana. Bogotá: Ascofade.

Telias, A. (2010). Educación para la ciudadanía y Educación Ambiental: Una articulación necesaria". Memorias Congreso Iberoamericano de Educación. Metas 2021, Buenos Aires. Recuperado de http:// www.adeepra.org.ar/congresos/Congreso $\% 20$ IBEROAMERICANO/EDUCCIUDADANIA/ R1532_Aldana.pdf

Torres Carrasco, M. (2008). La Educación Ambiental: una estrategia fexible, un proceso y unos propósitos en permanente construcción. La experiencia de Colombia. Revista Iberoamericana de Educación: Educación Ambiental y Formación: Proyectos y Experiencias, (16), 23-27. Recuperado de http://www.rieoei.org/oeivirt/ rie16a02.pdf 
